\title{
ON A PULSATILE FLOW OF A TWO-PHASE VISCOUS FLUID IN A TUBE OF ELLIPTIC CROSS-SECTION
}

\author{
A.K. GHOSH \\ Department of Mathematics \\ Jadavpur University \\ Calcutta 700032, India \\ A.R. KHAN \\ Department of Mathematics \\ Taki Government College \\ 24 - Parganas, West Bengal \\ India \\ L. DEBNATH \\ Department of Mathematics \\ University of Central Florida \\ orlando, Florida 32816, U.S.A.
}

(Received December 2, 1988 and in revised form May 2, 1989)

ABSTRACT. A study is made of an unsteady flow of an incompressible viscous fluid with embedded small inert spherical particles contained in a tube of elliptic cross-section due to a periodic pressure gradient acting along the length of the tube. The solutions for the fluid velocity and the particle velocity are obtained for large and small times. It is shown that the effect of particles on the flow is significant in the small-time solution while the large-time solution shows no effect of the particles on the flow.

\section{INTRODUCTION.}

Considerable attention has been given to pulsatile flows of fluids in a tube of various cross-section due to its increasing importance in the study of blood flow in arteries. Womersley (1955) has studied the pulsatile flow of a viscous fluid in a tube of circular cross-section due to a given pressure gradient. Similar problems have been investigated for the unsteady flow of a viscoelastic liquid by Walters and King (1970 - 1971). Khamrui (1955) has obtained solutions for a periodic flow of a viscous liquid in a tube of elliptic section under the influence of a periodic pressure gradient. Later on, Ghosh and Khamrui (1978) have investigated the pulsatile flow model of a viscoelastic fluid in a channel of elliptic cross-section. In spite of these works, there seems to be no study of pulsatile flow of a two-phase viscous liquid in a tube of elliptic section due to a periodic pressure gradient. The main objective of this paper is to investigate such problem in order to determine the fluid velocity as well as the particle velocity, and to examine the effects of particles on 
the flow. It is shown that the effect of particles on the flow is significant in the small-time solution while the largettime snlution contains no effect of particles on the flow.

\section{MATHEMATICAi FORIJMULATION.}

Based upon the Saffman (1962) two-phase fluid model, the equations of unsteady motion of an incompressible viscous fluid with embedded identical sinall inert spherical particles are

$$
\begin{aligned}
& \frac{\partial u}{\partial t}=-\frac{1}{\rho} \frac{\partial \rho}{\partial z}+v\left(\frac{\partial^{2} u}{\partial x^{2}}+\frac{\partial^{2} u}{\partial y^{2}}\right)+\frac{k}{\tau}(v-u) \\
& \frac{\partial v}{\partial t}=\frac{1}{\tau}(u-v)
\end{aligned}
$$

where $u, v$ are the components of the fluid and the particle velocity in the direction of z-axis which is taken along the length of the tube. The last term on the right hand side of equation (2.1) represents the force exerted by the particles on the flow while the term on the right hand side of equation (2.2) is a simllar force-term exerted by the fluid on the particles. $k=\frac{\mathrm{mN}_{0}}{\rho}$ is the ratio of the mass density of the particles and the fluid, commonly known as mass concentration of the dust particles. $\tau=\frac{m}{k}$ is the relaxation time of the particles. $m, N, k, \rho$ and $\nu$ are respectively the mass of a particle, the number density of particles, the Stokes resistance coefficient, the density and the kinematic viscosity of the fluid.

The flow is generated from rest due to the periodic pressure gradient acting along the length of the tube as

$$
\frac{\partial p}{\partial z}=P e^{i \omega t}
$$

where $P$ is a constant and $\omega$ is the frequency.

The initial conditions are $u(x, y, 0)=0$ and $v(x, y, 0)=0$

\section{THE LAPLACE TRANSFORMED SOLUTION.}

We apply the Laplace transform of $u(x, y, t)$ and $v(x, y, t)$ with respect to $t$ defined by (Myint-U and Debnath [1987])

$$
\bar{u}(x, y, s)=\int_{0}^{\infty} e^{-s t} u(x, y, t) d t
$$

to solve the differential system (2.1) - (2.3). The transformed equation for $\bar{u}(x, y, s)$ is given by

$$
\frac{\partial^{2} u}{\partial x^{2}}+\frac{\partial^{2} \bar{u}}{\partial y^{2}}-\frac{s}{v}\left(\frac{1+k+s \tau}{1+s \tau}\right) \bar{u}=\frac{P}{\rho \nu} \frac{1}{(s-1 \omega)}
$$


Substituting $\vec{u}=\bar{U}-\frac{P(1+s \tau)}{\rho s(s-i \omega)(\overline{l+k}+s \tau)}$ into (3.2), we obtain

$$
\frac{\partial^{2} \bar{U}}{\partial x^{2}}+\frac{\partial^{2} \bar{U}}{\partial y^{2}}-q^{2} \bar{U}=0
$$

where

$$
q^{2}=\frac{s(1+k+s \tau)}{v(1+s \tau)}
$$

We next introduce the elliptic coordinate $(\xi, \eta)$ defined by $x+i y=c \cosh (\xi+i \eta)$ where $c=\left(a^{2}-b^{2}\right)^{1 / 2}$ in (3.3) to transform equation (3.3) in the form

$$
\frac{\partial^{2} \bar{U}}{\partial \xi^{2}}+\frac{\partial^{2} \bar{U}}{\partial \eta^{2}}-2 \ell^{2}(\cosh 2 \xi-\cos 2 \eta) \bar{U}=0
$$

where $\quad 4 \ell^{2}=q^{2} c^{2}$.

Separating the variables by using $\bar{U}=\bar{\phi}(\xi) \bar{\psi}(n)$, we find a modified Mathieu equation for $\bar{\phi}(\xi)$, and a Mathieu equation for $\bar{\psi}(\eta)$ as.

$$
\begin{aligned}
& \frac{\partial^{2} \bar{\phi}}{\partial \xi^{2}}-\left(a_{1}-2 \ell^{2} \cosh 2 \xi\right) \bar{\phi}=0 \\
& \frac{\partial^{2} \bar{\psi}}{\partial \eta^{2}}+\left(a_{1}+2 \ell^{2} \cos 2 \eta\right) \bar{\psi}=0
\end{aligned}
$$

where $a_{1}$ is a constant.

Since $\bar{U}$ is symmetrical with respect to the axis of the ellipse and is periodic with period $\pi, \bar{\psi}$ is a periodic function ${ }_{2 n}\left(n, l^{2}\right)$ of order $2 n$ (see Mclachlan (1947)). $\bar{\phi}$ is then the modified Mathieu function $\mathrm{Ce}_{2 \mathrm{n}}\left(\xi,-\ell^{2}\right)$. these are represented by expansions

$$
\begin{aligned}
& \operatorname{ce}_{2 n}\left(n,-l^{2}\right)=(-1)^{n} \sum_{r=0}^{\infty}(-1)^{r} A_{2 r}^{(2 n)} \cos 2 r n, \\
& \operatorname{Ce}_{2 n}\left(\xi,-l^{2}\right)=(-1)^{n} \sum_{r=0}^{\infty}(-1)^{r} A_{2 r}^{(2 n)} \cosh 2 r \xi,
\end{aligned}
$$

where $A_{2 r}^{(2 n)}$ are functions of $\ell^{2}$.

The appropriate general solution for $\bar{u}$ then becomes

$$
\bar{u}=-\frac{p}{\rho} \frac{1+s \tau}{s(s-i \omega)(1+k+s \tau)}+\sum_{r=0}^{\infty} C_{2 n} C e_{2 n}\left(\xi,-\ell^{2}\right) c e_{2 n}\left(n,-l^{2}\right) .
$$

If $\xi=\xi_{0}$ designates the boundary of the ellipse, the boundary condition reads

$$
\bar{u}=0 \quad \text { when } \xi=\xi_{0}
$$

That is,

$$
\frac{p}{\rho} \frac{(1+s \tau)}{s(s-1 \omega)(1+k+s \tau)}=\sum_{r=0}^{\infty} C_{2 n} C e_{2 n}\left(\xi_{0},-l^{2}\right) C_{2 n}\left(n,-\ell^{2}\right) .
$$


Multiplying (3.11) by $\operatorname{ce}_{2 n}\left(n,-\ell^{2}\right)$ and integrating from 0 to $2 \pi$ with respect to $n$ and then using orthogonal relations, we obtain

$$
C_{2 n}=\frac{2 \pi(-1)^{n_{A}}(2 n)}{C e_{2 n}\left(\xi_{0},-\ell^{2}\right) I_{2 n}} \cdot \frac{P(1+s \tau)}{\rho s(s-i \omega)(1+k+s \tau)}
$$

where

$$
I_{2 n}=\int_{0}^{2 \pi} c e_{2 n}\left(n,-\ell^{2}\right) d n
$$

Hence

$$
\bar{u}=-\frac{P}{\rho} \frac{1+s \tau}{s(s-i \omega)(1+k+s \tau)}+\sum_{r=0}^{\infty} C_{2 n} C e_{2 n}\left(\xi,-\ell^{2}\right) c e{ }_{2 n}\left(n,-\ell^{2}\right)
$$

with $\quad B=\ell^{2}=\frac{q^{2} c^{2}}{4}$.

4. SOLUTIONS FOR SMALL AND LARGE TIMES.

The form of $q$, that is, $\ell$ clearly suggests that the Laplace inversion of (3.14) is almost a formidable task. So in order to give a fairly good description of the flow, the inversion will be considered in two limiting cases of small and large values of $B\left(=\ell^{2}\right)$.

Case I: For small values of $B$, we have

$$
\begin{aligned}
& \operatorname{ce}_{0}(n,-\beta) \simeq\left(1+\frac{1}{2} \beta \cos 2 n\right) \\
& \operatorname{ce}_{2}(n,-\beta) \simeq \cos 2 n+\beta\left(\frac{1}{12} \cos 4 n-\frac{1}{4}\right)
\end{aligned}
$$

and similar asymptotic expansions for the modified Mathieu functions.

Also from Mclachalan (1947), we get

$$
A_{0}^{(0)}=1, A_{0}^{(2)}=\frac{1}{4} B+O\left(B^{3}\right), A_{0}^{(2 n)}=O\left(B^{n}\right)
$$

so that

$$
\begin{aligned}
& C_{0}=\frac{P}{\rho} \frac{(1+s \tau)}{s(s-1 \omega)(1+k+s \tau)}\left(1-\frac{1}{2} \beta \cosh 2 \xi_{0}\right) \\
& C_{2}=-\frac{1}{2} \frac{P(1+s \tau)}{\rho s(s-i \omega)(1+k+s \tau)} \cdot \frac{B}{\cosh 2 \xi_{0}}
\end{aligned}
$$

Substituting these is (3.14), we obtain

$$
\bar{u}=-\frac{p c^{2}}{8 n_{0}(8-1 \omega)}\left[\cosh 2 \xi_{0}-\cosh 2 \xi-\cos 2 n+\frac{\cosh 2 \xi \cos 2 n}{\cosh 2 \xi_{0}}\right]
$$

where $\eta_{0}$ is the viscosity of the fluid. 
The inversion of $(4.5)$ gives

$$
u=-\frac{P}{8} c^{2} e^{i \omega t}\left[\cosh 2 \xi_{0}-\cosh 2 \xi-\cos 2 n+\frac{\cosh 2 \xi \cos 2 \eta}{\cosh 2 \xi_{0}}\right]
$$

Cons equent ly

$$
\operatorname{Re}\{u\}=-\frac{P}{8} \frac{c^{2}}{\eta_{0}}\left[\cosh 2 \xi_{0}-\cosh 2 \xi-\cos 2 \eta+\frac{\cosh 2 \frac{2}{\cosh } 2 \xi}{2 \xi} \frac{\cos 2 \eta}{0}\right] \cos \omega t
$$

This result describes the fluid velocity for small $B$ (or small $s$ ), that is for large time $t$. Thus the large time solution does not show any effect of the particles on the fluid flow.

The particle velocity in this case can be obtained by integrating (2.2) and has the form

$$
v=-\frac{P c^{2}}{8 \eta_{0}} \frac{e^{i \omega t}-e^{-t / \tau}}{1+i \omega \tau}\left[\cosh 2 \xi_{0}-\cosh 2 \xi-\cos 2 n+\frac{\cosh 2 \xi \cos 2 \eta_{0}}{\cosh 2 \xi_{0}}\right]
$$

and its real part is

$$
\operatorname{Re}\{v\}=-\frac{P c^{2}}{8 \eta_{0}} \frac{\left(\cos \omega t+\omega \tau \sin \omega t-e^{-t / \tau}\right)}{1+\omega^{2} \tau^{2}}\left[\cosh 2 \xi_{0}-\cosh 2 \xi-\cos 2 n+\frac{\cosh 2 \xi \cos 2 \eta}{\cosh 2 \xi_{0}}\right]
$$

Expressing in Cartesian form of (4.7) and (4.9), we obtain

$$
\begin{aligned}
& \operatorname{Re}\{u\}=-\frac{P}{2 \eta_{0}}\left(\frac{a^{2} b^{2}}{a^{2}+b^{2}}\right) \cos \omega t\left(1-\frac{x^{2}}{a^{2}}-\frac{y^{2}}{b^{2}}\right), \\
& \operatorname{Re}(v)=-\frac{P}{2 n_{0}}\left(\frac{a^{2} b^{2}}{a^{2}+b^{2}}\right) \frac{\cos \omega t+\omega \tau \sin \omega t}{1+\omega^{2} \tau^{2}}-e^{-t / \tau}\left(1-\frac{x^{2}}{a^{2}}-\frac{y^{2}}{b^{2}}\right)
\end{aligned}
$$

These results indicate that in the limit $t+\infty$, the fluid moves faster than the particles with a phase lead $\tan ^{-1} \omega \tau$ if $\omega \neq 0$, and for $\omega=0$, the fluid and the particle move in unision in the ultimate steady state condition.

Case II: When $B$ is large (or $t$ is smal1), it follows from Mclachlan (1947) that $A_{0}^{(0)}=1$ and for $n>1,\left|A_{0}{ }^{(2 n)}\right|$ is very small. Consequently, the asymptotic formula gives

$$
\mathrm{Ce}_{0}(\xi,-\beta) \simeq\left(\frac{2}{1 \sinh \xi}\right) K_{0} \cosh \left[2 l \cosh \xi-\tanh ^{-1} \tan (\pi / 4-1 \xi / 2)\right]
$$

where $\quad \mathrm{K}_{0}=\frac{\mathrm{Ce}_{0}(0) \mathrm{Ce}_{0}(\pi / 2)}{\mathrm{A}_{0}^{(0)}(2 \pi l)^{1 / 2}}$.

Since $2 \ell=q c$, we get

$$
\begin{aligned}
& \cosh \left[2 l \cosh \xi-\tanh ^{-1}\left\{\tan \left(\frac{\pi}{4}-\frac{1 \xi}{2}\right)\right\}\right] \\
& \simeq \frac{1}{2} \exp \left\{q c \cosh \xi-\tanh ^{-1} \tan \left(\frac{\pi}{4}-\frac{1 \xi}{2}\right)\right\}=\frac{1}{2}\left(\tanh \frac{1}{2} \xi\right)^{1 / 2} \exp \{q c \cosh \xi\} .
\end{aligned}
$$


Hence for large values of $B$ i.e. for large $|q|$,

$$
\bar{u}=-\frac{p}{\rho} \frac{(1+s \tau)}{s(s-1 \omega)(1+k+s \tau)}+\frac{p}{\rho} \frac{1+s \tau}{s(s-i \omega)(1+k+s \tau)} \frac{\cosh \frac{1}{2} \xi_{0}}{\cosh \frac{1}{2} \xi} \exp \left\{-q c\left(\cosh \xi_{0}-\cosh \xi\right)\right\} .
$$

The inversion of $(4.14)$ yields

$$
\begin{aligned}
& \operatorname{Re}\{u\}=-\frac{P}{\rho \omega\left[(1+k)^{2}+\omega^{2} \tau^{2}\right]}\left\{\omega \tau k \cos \omega t+\left(1+k+\omega^{2} \tau^{2}\right) \sin \omega t-\omega \tau k e^{-\frac{1+k}{t}}\right\} \\
& +\frac{P}{\rho \omega\left[(1+k)^{2}+\omega^{2} \tau^{2}\right]}\left\{e^{-r}\left[\omega \tau k \cos (\omega t-\delta)+\left(1+k+\omega^{2} \tau^{2}\right) \sin (\omega t-\delta)\right]\right.
\end{aligned}
$$

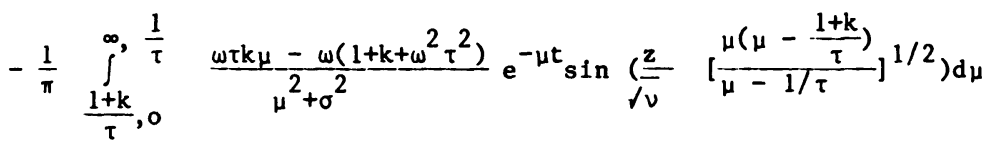

$$
\begin{aligned}
& \left.+\frac{\omega k}{\pi} \tau \int_{\frac{1+k}{\tau}, 0}^{\infty} \frac{1}{\tau} \frac{e^{-\mu t}}{\mu-\frac{1+k}{\tau}} \sin \left(\frac{z}{\tau \nu}\left[\frac{\mu\left(\mu-\frac{1+k}{\tau}\right.}{\mu-\frac{1}{\tau}}\right]^{1 / 2}\right) d \mu\right\} \frac{\cosh \frac{1}{2} \xi_{0}}{\cosh \frac{1}{2} \xi}
\end{aligned}
$$

where

$$
\begin{aligned}
& r=z \alpha_{1}\left(\frac{\omega}{2 \nu\left(1+\omega^{2} \tau^{2}\right)}\right)^{1 / 2}, \delta=z \alpha_{2}\left(\frac{\omega}{2 \nu\left(1+\omega^{2} \tau^{2}\right)}\right)^{1 / 2}, z=c\left(\cosh \xi_{0}-\cosh \xi\right), \\
& \alpha_{1}, \alpha_{2}=\left(\frac{\omega}{2}\right)^{1 / 2}\left\{\left[\omega^{2} k^{2} \tau^{2}+\left(1+k+\omega^{2} \tau^{2}\right)^{2}\right]^{1 / 2} \pm \omega \tau k\right\}^{1 / 2} \text { and } \int_{c, e}^{d, f}=\int_{c}^{d}+\int_{e}^{f} .
\end{aligned}
$$

In the case of fluid flow without particles $(k=0)$, the velocity field (4.15) assumes the form

$$
\begin{aligned}
& \operatorname{Re}\{u\}=\frac{P}{\rho}-\frac{\sin \omega t}{\omega}+\frac{P}{\rho \omega}\left\{\sin \left[\omega t-\sqrt{\frac{\omega}{2 \nu}} c\left(\cosh \xi_{0}-\cosh \xi\right)\right] \times\right. \\
& \left.\times \exp \left[-\sqrt{\frac{\omega}{2 \nu}} c\left(\cosh \xi_{0}-\cosh \xi\right)\right]\right\} \frac{\cosh 1 / 2 \xi_{0}}{\cosh \frac{1}{2} \xi}+ \\
& +\frac{P}{\pi \rho} \frac{\cosh \frac{1}{2} \xi_{0}}{\cosh \frac{1}{2} \xi} \int_{0}^{\infty} \frac{e^{-\mu t}}{\mu^{2}+\omega^{2}} \sin \left[c\left(\cosh \xi_{0}-\cosh \xi\right) \sqrt{ } \frac{\bar{\mu}}{\nu}\right] d \mu
\end{aligned}
$$

This is identical with Khamrui's result (1955), when $P$ is replaced by $-P$ and $t+\infty$. We further note that the result (4.15) represents the small-time solution for the twophase fluid velocity. Moreover, the presence of the parameter $k$ and $\tau$ in (4.15) indicates that the fluid velocity is significantly affected by the particles when $t$ is very small. This small-time solution also exhibits the boundary layer character of the flow similar to that of the fluid motion without particles. The thickness of the boundary layer decreases with increasing values of the particle concentration. 
The particle velocity, when $\beta$ is large, is given by

$$
\begin{aligned}
& \operatorname{Re}\{v\}=-\frac{P}{\rho \omega\left[(1+k)^{2}+\omega^{2} \tau^{2}\right]}-\left\{\omega \tau k\left[\frac{\tau \omega \sin \omega t+\cos \omega t-e^{-t / \tau}}{1+\tau^{2} \omega^{2}}\right]+\right. \\
& \left.+\left(1+k+\omega^{2} \tau^{2}\right)\left[\frac{\omega \tau e^{-t / \tau} \sin \omega t-\omega \tau \cos \omega t}{1+\tau^{2} \omega^{2}}\right]-\omega \tau\left(e^{-t / \tau}-e^{-\frac{1+k}{\tau}}\right)\right\} \\
& +\frac{P}{\rho \omega\left[(1+k)^{2}+\omega^{2} \tau^{2}\right]} \frac{\cosh \frac{1}{2} \xi_{0}}{\cosh \frac{1}{2} \xi}\left[e ^ { - r } \left\{\omega \tau k\left[\frac{\tau \omega \sin (\omega t-\delta)+\cos (\omega t-\delta)+e^{-t / \tau}(\omega \tau \sin \delta-\cos \delta)}{1+\omega^{2} \tau^{2}}\right]++\right.\right. \\
& \left.+\left(1+k+\omega^{2} \tau^{2}\right)\left[\frac{\sin (\omega t-\delta)-\omega \tau \cos (\omega t-\delta)+e^{-t / \tau}(\omega \tau \cos \delta+\sin \delta)}{1+\omega^{2} \tau^{2}}\right]\right\} \\
& -\frac{1}{\pi} \int_{\frac{1+k}{\tau}, 0}^{\infty, \frac{1}{\tau}} \frac{\omega \tau k \mu-\omega\left(1+k+\omega^{2} \tau^{2}\right)}{\tau(\mu-1 / \tau)\left(\mu^{2}+\omega^{2}\right)}\left(e^{-t / \tau}-e^{-\mu t}\right) \sin \left[\frac{z}{\sqrt{\nu}}\left[\frac{\mu\left(\mu-\frac{1+k}{\tau}\right)}{\mu-\frac{1}{\tau}}\right]^{1 / 2}\right\} d \mu \\
& \left.+\frac{\omega k}{\pi} \int_{\frac{1+k}{\tau}, 0}^{\infty, \frac{1}{\tau}} \frac{e^{-t / \tau}-e^{-\mu t}}{(\mu-1 / \tau)\left(\mu-\frac{1+k)}{\tau}\right.} \sin \left\{\frac{z}{\nu \nu}\left[\frac{\mu\left(\mu-\frac{1+k}{\tau}\right)}{\mu-1 / \tau}\right] 1 / 2\right\} d \mu\right]
\end{aligned}
$$

Finally, the results corresponding to the circular cylinder are obtained by replacing $c\left(\cosh \xi_{0}-\cosh \xi\right)$ by $(a-r)$ and $\frac{\cosh \frac{1}{2} \xi_{0}}{\cosh \frac{1}{2} \xi}$ by $\gamma \frac{a}{r}$ in (4.15) and (4.17) where a is the radius of the cylinder. In particular, when $k=0$, we find from (4.16) that

$$
\begin{aligned}
\operatorname{Re}\{u\} & =-\frac{P}{\rho} \frac{\sin \omega t}{\omega}+\frac{P}{\rho}\left(\frac{a}{r}\right)^{1 / 2}\left[\frac{1}{\omega} \sin \left\{\omega t-(a-r) \sqrt{\frac{\omega}{2 \nu}}\right\}\right. \\
& \left.+\frac{1}{\pi} \int_{0}^{\infty} \frac{e^{-\mu t}}{\mu^{2}+\omega^{2}} \sin \left\{(a-r) \frac{j}{\nu}\right\} d \mu\right] .
\end{aligned}
$$

where $r^{2}=x^{2}+y^{2}$. This is a well-known result for large $\omega$ and $t+\infty$.

\section{REFERENCES}

1. WOMERSLEY, J.R., Method for the calculation of velocity, Rate of flow and viscous drag in arteries when the pressure gradient is known, J. Physiol. 127 (1955), 553.

2. WALTERS, N.D. and KING, M.J., Unsteady flow of an elastico-viscous 1iquid, Rheol. Acta. 99 (1970), 345-355. 
3. WALTERS, N.D. and KING, M.J., The unsteady flow of an elastico-viscous liquid in a straight pipe of circilar cross section, J. Phys. D: Appl. Phys. 4 (1971), 204-211.

4. KHAMRUI, S.R., On the flow of a viscous liquid through a tube of elliptic section under the influence of a periodic pressure gradient, Bull. Cal. Math.Soc. 49 (1955), 57-60.

5. GHOSH, A.K. and KHAMRUI, S.K., Pulsatile flow of an elastlco-Viscous liquid in a channel of elliptic cross section, Rheol. Acta. 17 (1978), 227-230.

6. SAFFMAN, P.G., On the Stability of Laminar flow of a dusty gas, J. Fluid Mech. $13(1962), 120-128$.

7. McLACHLAN, N.W., Theory and Application of Mathieu Functions Oxford University Press, (1947). 


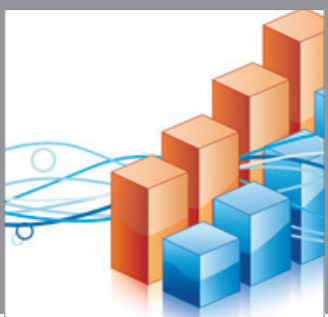

Advances in

Operations Research

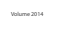

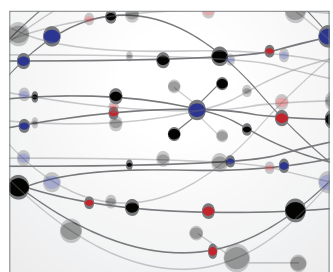

\section{The Scientific} World Journal
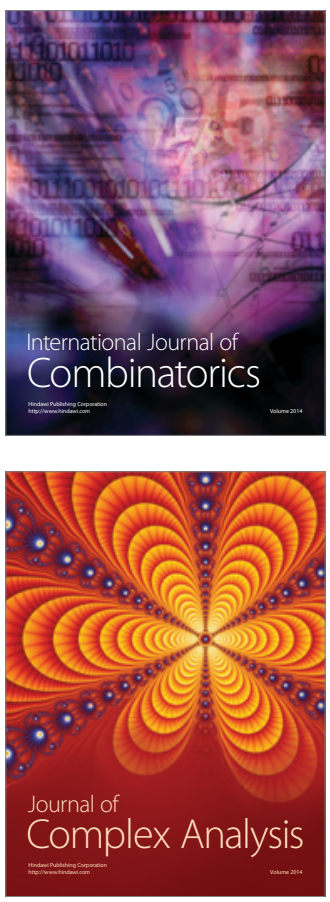

International Journal of

Mathematics and

Mathematical

Sciences
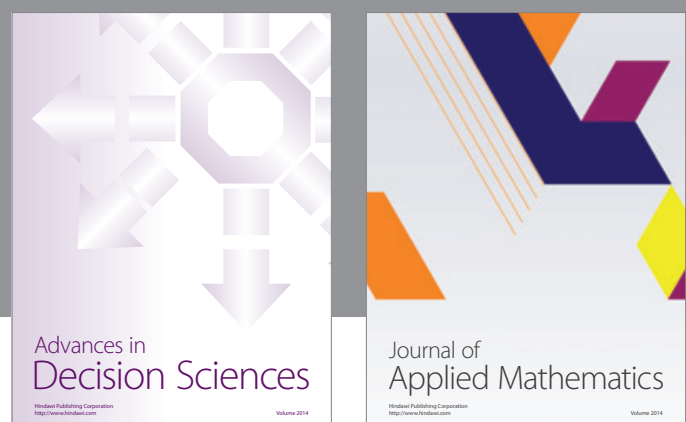

Journal of

Applied Mathematics
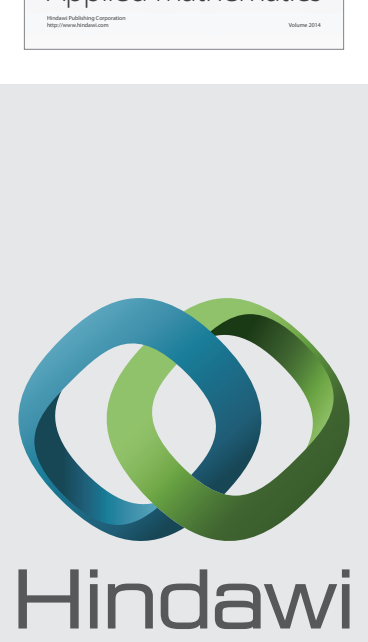

Submit your manuscripts at http://www.hindawi.com
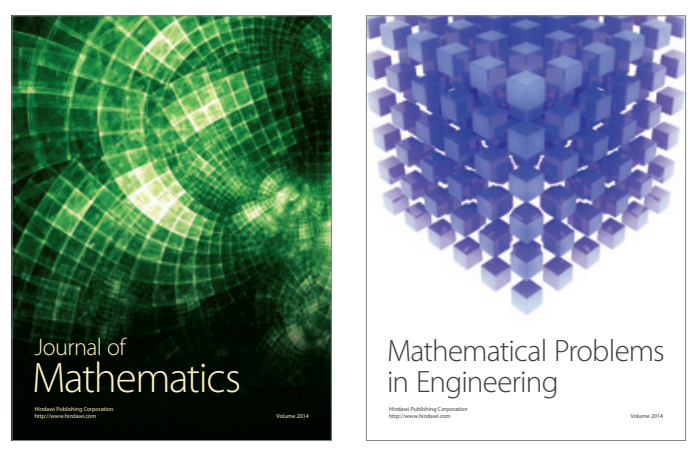

Mathematical Problems in Engineering
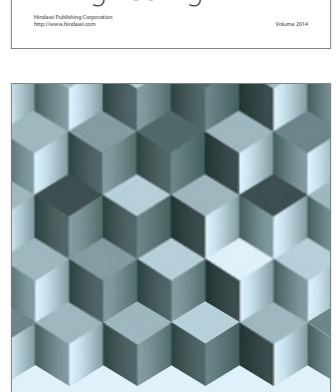

Journal of

Function Spaces
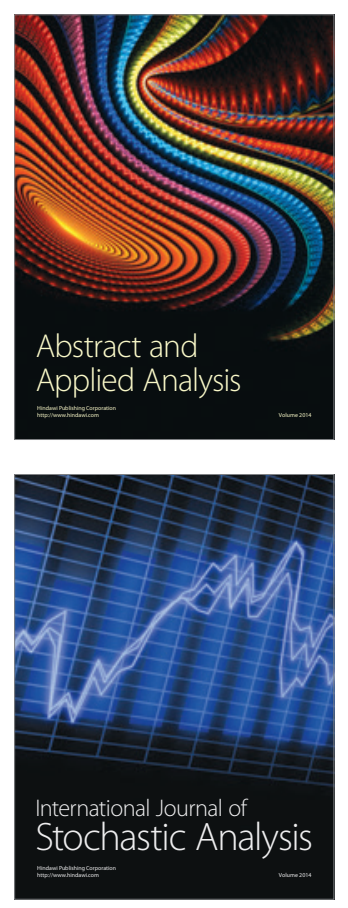

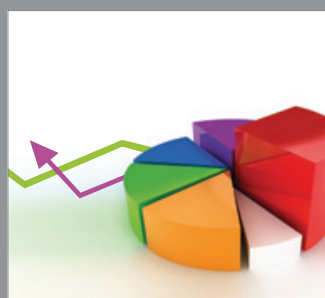

ournal of

Probability and Statistics

Promensencen
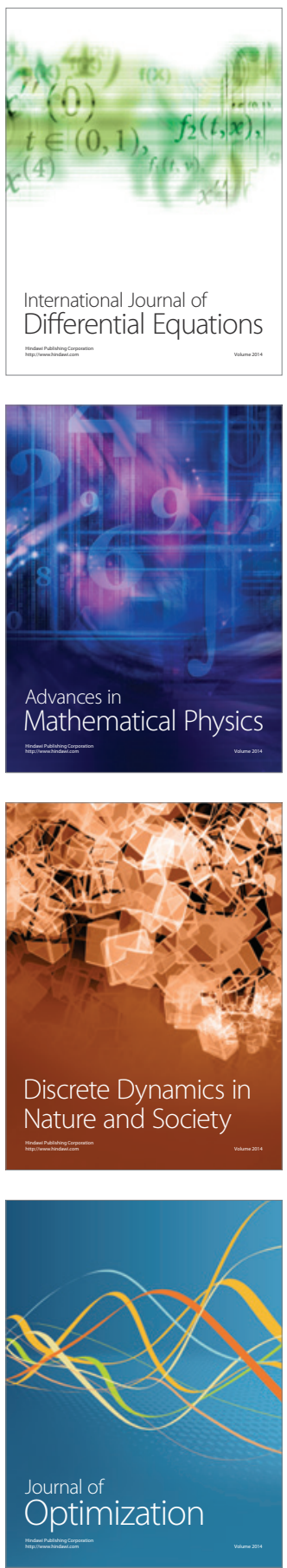